\title{
Local Projection-Based Stabilized Mixed Finite Element Methods for Kirchhoff Plate Bending Problems
}

\author{
Xuehai Huang \\ College of Mathematics and Information Science, Wenzhou University, Wenzhou 325035, China \\ Correspondence should be addressed to Xuehai Huang; xuehaihuang@gmail.com
}

Received 5 December 2012; Accepted 18 February 2013

Academic Editor: Carlos Vazquez

Copyright (C) 2013 Xuehai Huang. This is an open access article distributed under the Creative Commons Attribution License, which permits unrestricted use, distribution, and reproduction in any medium, provided the original work is properly cited.

\begin{abstract}
Based on stress-deflection variational formulation, we propose a family of local projection-based stabilized mixed finite element methods for Kirchhoff plate bending problems. According to the error equations, we obtain the error estimates of the approximation to stress tensor in energy norm. And by duality argument, error estimates of the approximation to deflection in $H^{1}$-norm are achieved. Then we design an a posteriori error estimator which is closely related to the equilibrium equation, constitutive equation, and nonconformity of the finite element spaces. With the help of Zienkiewicz-Guzmán-Neilan element spaces, we prove the reliability of the a posteriori error estimator. And the efficiency of the a posteriori error estimator is proved by standard bubble function argument.
\end{abstract}

\section{Introduction}

To design conforming finite element method for fourthorder elliptic partial differential equation, it requires $C^{1}$ continuity finite element space which is arduous to construct (cf. [1]). Alternatively, mixed finite element methods are preferred because $C^{0}$-continuity finite element space is sufficient for deflection. Another advantage of mixed finite element methods is that the stress or $\Delta u$ can be approximated simultaneously.

One kind of mixed finite element methods is based on Ciarlet-Raviart method whose unknowns are $\Delta u$ and deflection $u$ (cf. [2]). Optimal convergence rate of the approximation to $u$ and suboptimal convergence rate of the approximation to $\Delta u$ of Ciarlet-Raviart method were obtained in [3-5], and a posteriori error analysis was given by [6]. It is worth to mention that mixed discontinuous Galerkin method for biharmonic equation advanced in [7] is on the basis of Ciarlet-Raviart method. Based on a first-order system and using single face-hybridizable technique in [8], Cockburn et al. derived a hybridizable and superconvergent DG method in [9] which improved the convergence rate of the approximation to $\Delta u$.

Another kind of mixed finite element methods for Kirchhoff plate bending problems is based on stress-deflection formulation. Standard stress-deflection mixed finite element methods require the finite element space for stress belonging to $\mathbf{H}(\operatorname{div}, \Omega, \mathbb{S})$, which is substantially difficult to construct since the tensor-valued function must be symmetric and belong to $\mathbf{H}(\operatorname{div}, \Omega)$ simultaneously. As far as we know, the only standard mixed finite element method of this kind mentioned in [10] adopts composite element. Fortunately, several $\mathbf{H}(\operatorname{div}, \Omega, \mathbb{S})$-conforming elements have been developed in the last decade. Arnold and Winther designed the first pure polynomial $\mathbf{H}(\operatorname{div}, \Omega, \mathbb{S})$-conforming elements in two dimensions in [11], which were extended to three dimensions in $[12,13]$. The vertex degrees of freedom are unavoidable when using pure polynomial shape function spaces, which is demonstrated in [11]. With regard to this, Guzmán and Neilan constructed $\mathbf{H}(\operatorname{div}, \Omega, \mathbb{S})$-conforming elements by enriching the polynomial shape function spaces with rational bubble functions which can avoid vertex degrees of freedom in [14]. On the other hand, some efforts have been made to lower the requirement of $\mathbf{H}(\operatorname{div}, \Omega, \mathbb{S})$-conforming finite element space for stress. Along this way, Hellan-Herrmann-Johnson method raised in [15-17] is a wonderful mixed method for plate bending problems whose convergence rates for both variables are optimal. Behrens and Guzmán introduced a new mixed method which is based on a system of firstorder equations and uses nonsymmetric finite element tensor 
space to approximate stress in [18]. And a hybrid technique is used for this mixed method to reduce the globally coupled degrees of freedom to only those associated with Lagrange multipliers, which is very efficient in implementation. Moreover, a local postprocessing technique is used to produce new approximation of $u$ with superconvergence rate. In the context of DG methods, LCDG method using fully discontinuous finite element space for stress devised in [19] is also based on stress-deflection formulation.

In this paper, we propose a family of local projectionbased stabilized mixed finite element methods for problem (1) based on the stress-deflection variational formulation. The stress tensor will be approximated in Arnold-Winther element spaces [11] which uses polynomial shape functions, and deflection will be approximated in Lagrangian element spaces $[1,20]$ in our mixed methods. To ensure the well-posedness of our mixed methods, we use local projection method which has been widely used in Stokes equation (cf. [21, 22]), secondorder elliptic problems (cf. [23-25]), and fourth-order obstacle problem (cf. [26]). According to the error equations, we obtain that the convergence rate of the approximation to stress tensor in energy norm is $O\left(h^{\min \{m-1+\alpha, k-1-\alpha\}}\right)$. And by duality argument, the convergence rate of the approximation to deflection in $H^{1}$-norm is shown to be $O\left(h^{\min \{m-1+2 \alpha, k-1\}}\right)$. An error estimator is proposed which is closely related to the second-order system (equilibrium equation and constitutive equation) and nonconformity of the finite element spaces. Using the similar argument as in [6] by replacing Hsieh-Clough-Tocher element space by ZienkiewiczGuzmán-Neilan element space proposed in [27], we prove the reliability of the a posteriori error estimator with all orders. However, by using Hsieh-Clough-Tocher element space which includes the third-order polynomials, the a posteriori error estimator is only proved to be reliable for $k=2,3$ in [6]. Furthermore, efficiency of the a posteriori error estimator is achieved by bubble function argument.

In the end of this section, let us describe the Kirchhoff plate bending problem. Assume that a thin plate occupies a bounded polygonal domain $\Omega \subset \mathbb{R}^{2}$. The mathematical model of this plate clamped on the boundary under a vertical load $f \in L^{2}(\Omega)$ is governed by (cf. $[28,29]$ )

$$
\begin{array}{lc}
\mathscr{C}^{-1} \boldsymbol{\sigma}=\mathscr{K}(u) \quad \text { in } \Omega, \\
\nabla \cdot(\nabla \cdot \boldsymbol{\sigma})=-f \quad \text { in } \Omega, \\
u=\partial_{\mathbf{n}} u=0 \quad \text { on } \partial \Omega,
\end{array}
$$

where $\mathbf{n}$ is the unit outward normal to $\partial \Omega, \boldsymbol{\nabla}$ is the usual gradient operator, $\nabla \cdot$ stands for the divergence operator acting on tensor-valued or vector-valued functions (cf. [29]), and

$$
\begin{gathered}
\sigma:=(1-v) \mathscr{K}(u)+v \operatorname{tr}(\mathscr{K}(u)) \mathscr{I}, \\
\mathscr{K}(u):=\left(\mathscr{K}_{i j}(u)\right)_{2 \times 2}, \\
\mathscr{K}_{i j}(u):=-\partial_{i j} u, \quad 1 \leq i, j \leq 2,
\end{gathered}
$$

with $\mathscr{I}$ a second-order identity tensor, tr the trace operator acting on second order tensors, and $v \in L^{\infty}(\Omega)$ the Poisson ratio satisfying inf $\operatorname{in}_{x \in \Omega} \nu>0$ and $\sup _{x \in \Omega} \nu<0.5$. For simplicity hereinafter, we introduce a symmetric and positive definite operator $\mathscr{C}$ defined as follows: for any second-order tensor $\boldsymbol{\tau}$,

$$
\mathscr{C} \boldsymbol{\tau}:=(1-\nu) \tau+\nu \operatorname{tr}(\boldsymbol{\tau}) \mathscr{I}
$$

Then it is easy to see that $\boldsymbol{\sigma}=\mathscr{C} \mathscr{K}(u)$ and $\mathscr{C}^{-1} \boldsymbol{\tau}=(1 /(1-$ $\nu)) \boldsymbol{\tau}-\left(\nu /\left(1-\nu^{2}\right)\right)(\operatorname{tr} \boldsymbol{\tau}) \mathscr{I}$ for any second-order tensor $\boldsymbol{\tau}$. The stress-deflection mixed variational formulation of problem (1) given in [10] will be obtained as follows: find $(\sigma, u) \in$ $\Sigma \times V$ such that

$$
\begin{array}{ll}
a(\boldsymbol{\sigma}, \boldsymbol{\tau})+b(\boldsymbol{\tau}, u)=0 & \forall \boldsymbol{\tau} \in \boldsymbol{\Sigma}, \\
-b(\boldsymbol{\sigma}, v)=\int_{\Omega} f v d x & \forall v \in V,
\end{array}
$$

where $\boldsymbol{\Sigma}:=\mathbf{H}(\operatorname{div}, \Omega, \mathbb{S}), V:=H_{0}^{1}(\Omega)$, and

$$
\begin{array}{cl}
a(\boldsymbol{\sigma}, \boldsymbol{\tau}):=\int_{\Omega} \mathscr{C}^{-1} \boldsymbol{\sigma}: \boldsymbol{\tau} d x & \forall \boldsymbol{\sigma}, \boldsymbol{\tau} \in \boldsymbol{\Sigma}, \\
b(\boldsymbol{\tau}, v):=-\int_{\Omega}(\nabla \cdot \boldsymbol{\tau}) \cdot \nabla v d x & \forall \boldsymbol{\tau} \in \boldsymbol{\Sigma}, v \in V .
\end{array}
$$

Here, the symbol : denotes the double dot product operation of tensors.

The rest of this paper is organized as follows. A family of local projection based stabilized mixed finite element methods based on stress-deflection variational formulation for Kirchhoff plate bending problems is proposed in Section 2. An a priori error analysis and a posteriori error analysis for the stabilized mixed finite element methods are given in Sections 3 and 4, respectively.

\section{Stabilized Mixed Finite Element Methods}

We will define a family of local projection based stabilized mixed finite element methods for solving problem (1) based on the stress-deflection variational formulation (4)-(5) in this section. For this, we first introduce some notations frequently used later on. Denote the space of all symmetric $2 \times 2$ tensor by $\mathbb{S}$. Given a bounded domain $G \subset \mathbb{R}^{2}$ and a nonnegative integer $m$, let $H^{m}(G)$ be the usual Sobolev space of functions on $G$, and let $\mathbf{H}^{m}(G, \mathbb{S})$ be the usual Sobolev space of functions taking values in $\mathbb{S}$. The corresponding norm and seminorm are denoted, respectively, by $\|\cdot\|_{m, G}$ and $|\cdot|_{m, G}$. If $G$ is $\Omega$, we abbreviate them by $\|\cdot\|_{m}$ and $|\cdot|_{m}$, respectively. Let $H_{0}^{m}(G)$ be the closure of $C_{0}^{\infty}(G)$ with respect to the norm $\|\cdot\|_{m, G}$. We also denote by $\mathbf{H}(\operatorname{div}, G, \mathbb{S})$ the Sobolev space consisting of all $\mathbf{L}^{2}(G, \mathbb{S})$ functions whose divergence is square-integrable. For an integer $l \geq 0, P_{l}(G)$ stands for the set of all polynomials in $G$ with the total degree no more than $l$, and $\mathbf{P}_{l}(G, \mathbb{X})$ denotes the tensor or vector version of $P_{l}(G)$ for $\mathbb{X}$ being $\mathbb{S}$ or $\mathbb{R}^{2}$, respectively. For any vector field $\mathbf{v}$, the symmetric part of the gradient of $\mathbf{v}$ be given by

$$
\boldsymbol{\varepsilon}(\mathbf{v}):=\frac{1}{2}\left(\nabla \mathbf{v}+(\nabla \mathbf{v})^{T}\right)
$$

with $(\cdot)^{T}$ meaning the transpose of the second-order tensor. 
Let $\left\{\mathscr{T}_{h}\right\}_{h>0}$ be a regular family of triangulations of $\Omega$ (cf. $[1,20]) ; h:=\max _{K \in \mathscr{T}_{h}} h_{K}$ and $h_{K}:=\operatorname{diam}(K)$. Let $\mathscr{E}_{h}$ be the union of all edges of the triangulation $\mathscr{T}_{h}$ and $\mathscr{E}_{h}^{i}$ the union of all interior edges of the triangulation $\mathscr{T}_{h}$. For any $e \in \mathscr{E}_{h}$, denote by $h_{e}$ its length. Based on the triangulation $\mathscr{T}_{h}$, let the finite element spaces be given by

$$
\begin{gathered}
\boldsymbol{\Sigma}_{h}:=\left\{\boldsymbol{\tau} \in \boldsymbol{\Sigma}:\left.\boldsymbol{\tau}\right|_{K} \in \mathbf{P}_{k}(K, \mathbb{S}),\left.\boldsymbol{\nabla} \cdot \boldsymbol{\tau}\right|_{K}\right. \\
\left.\in \mathbf{P}_{k-2}\left(K, \mathbb{R}^{2}\right) \forall K \in \mathscr{T}_{h}\right\}, \\
V_{h}:=\left\{v \in H_{0}^{1}(\Omega):\left.\mathrm{v}\right|_{K} \in P_{k}(K) \forall K \in \mathscr{T}_{h}\right\},
\end{gathered}
$$

with $k \geq 3$. The so-called Arnold-Winther element space $\boldsymbol{\Sigma}_{h}$ was designed by Arnold and Winther [11], which is the first $\mathbf{H}(\operatorname{div}, \Omega, \mathbb{S})$-conforming finite element space with pure polynomials in two dimensions in history. For any $K \in \mathscr{T}_{h}$, the degrees of freedom for Arnold-Winther element space $\Sigma_{h}$ are given as follows [11]:

(i) the values of three components of $\boldsymbol{\tau}$ at each vertex of $K$,

(ii) the values of the moments of degree at most $k-2$ of $\tau \mathbf{n}$ on each edge $e$ of $K$,

(iii) the values of the moments $\int_{K} \tau: \varsigma d x$ for all $\varsigma \epsilon$ $\mathbf{N}_{k-2}(K)$,

where $\mathbf{N}_{k-2}(K):=\boldsymbol{\varepsilon}\left(\mathbf{P}_{k-2}\left(K, \mathbb{R}^{2}\right)\right)+\mathbf{M}_{k-2}(K)$ with

$$
\mathbf{M}_{k-2}(K):=\left\{\boldsymbol{\tau} \in \mathbf{P}_{k}(K, \mathbb{S}): \nabla \cdot \boldsymbol{\tau}=0 \text { and } \boldsymbol{\tau} \mathbf{n}=0 \text { on } \partial K\right\} \text {. }
$$

Given an integer $l \geq 0$, define the elementwise finite element spaces

$$
\begin{gathered}
X_{l}:=\left\{v \in L^{2}(\Omega):\left.v\right|_{K} \in P_{l}(K) \forall K \in \mathscr{T}_{h}\right\}, \\
\mathbf{X}_{l}:=X_{l} \times X_{l} .
\end{gathered}
$$

Denote by $Q_{l}: L^{2}(\Omega) \rightarrow X_{l}$ the elementwise $L^{2}$ orthogonal projection operator. And let $\mathbf{Q}_{l}$ be the vector version of $Q_{l}$. It follows from [11] that Arnold-Winther element space $\boldsymbol{\Sigma}_{h}$ has the following property:

$$
\nabla \cdot \Sigma_{h}=\mathbf{X}_{k-2} \cdot
$$

Now we can define a family of local projection based stabilized mixed finite element methods corresponding to the mixed formulation (4)-(5) for problem (1) as follows: find $\left(\boldsymbol{\sigma}_{h}, u_{h}\right) \in \boldsymbol{\Sigma}_{h} \times V_{h}$ such that

$$
\begin{gathered}
a\left(\boldsymbol{\sigma}_{h}, \boldsymbol{\tau}\right)+b\left(\boldsymbol{\tau}, u_{h}\right)=0 \forall \boldsymbol{\tau} \in \Sigma_{h}, \\
-b\left(\boldsymbol{\sigma}_{h}, v\right)+c\left(u_{h}, v\right)=\int_{\Omega} f v d x \forall v \in V_{h},
\end{gathered}
$$

where

$$
\begin{aligned}
& c(u, v) \\
& \quad:=\sum_{K \in \mathscr{T}_{h}} h_{K}^{-2 \alpha} \int_{K}\left(\nabla u-\mathbf{Q}_{k-2}(\nabla u)\right) \cdot\left(\nabla v-\mathbf{Q}_{k-2}(\nabla v)\right) d s
\end{aligned}
$$

with $0 \leq \alpha \leq 1$.
Next, let us illustrate the well-posedness of stabilized mixed finite element method (12)-(13).

Theorem 1. Stabilized mixed finite element methods (12)-(13) are uniquely solvable.

Proof. Since $\boldsymbol{\Sigma}_{h}$ and $V_{h}$ are both finite dimensional, it is enough to show that if $f=0$, then $\sigma_{h}=0, u_{h}=0$. Taking $\boldsymbol{\tau}=\boldsymbol{\sigma}_{h}$ in (12) and $v=u_{h}$ in (13), and adding two equalities, it follows that $a\left(\sigma_{h}, \boldsymbol{\sigma}_{h}\right)+c\left(u_{h}, u_{h}\right)=0$. Thus $\sigma_{h}=0$ and $\nabla u_{h}=\mathbf{Q}_{k-2}\left(\nabla u_{h}\right) \in \mathbf{X}_{k-2}$ in $\Omega$ from the definition of $a(\cdot, \cdot)$ and $c(\cdot, \cdot)$. Then (12) is reduced to

$$
b\left(\boldsymbol{\tau}, u_{h}\right)=-\int_{\Omega}(\nabla \cdot \boldsymbol{\tau}) \cdot \nabla u_{h} d x=0 \quad \forall \boldsymbol{\tau} \in \boldsymbol{\Sigma}_{h}
$$

According to (11), we get $\nabla u_{h}=0$, which means that $u_{h}$ is piecewise constant on $\mathscr{T}_{h}$. Therefore, $u_{h}=0$ for $u_{h} \in H_{0}^{1}(\Omega)$.

\section{A Priori Error Analysis}

In this section, we provide an a priori error analysis for stabilized mixed finite element methods (12)-(13).

3.1. Preliminaries. For a function $v \in L^{2}(\Omega)$ with $\left.v\right|_{K} \in$ $H^{m}(K)$ for all $K \in \mathscr{T}_{h}$, let $\|v\|_{m, h}$ and $|v|_{m, h}$ be the usual broken $H^{m}$-type norm and seminorm of $v$ :

$$
\begin{aligned}
\|v\|_{m, h} & =\left(\sum_{K \in \mathscr{T}_{h}}\|v\|_{m, K}^{2}\right)^{1 / 2}, \\
|v|_{m, h} & =\left(\sum_{K \in \mathscr{T}_{h}}|v|_{m, K}^{2}\right)^{1 / 2} .
\end{aligned}
$$

If $v$ is a vector-valued or tensor-valued function, the previous symbols are defined in the similar manners. For a vector or tensor $\mathbf{v}$, its length $|\mathbf{v}|$ is $(\mathbf{v} \cdot \mathbf{v})^{1 / 2}$ or $(\mathbf{v}: \mathbf{v})^{1 / 2}$. Moreover, define an energy norm for $\mathbf{L}^{2}(\Omega, \mathbb{S})$ and a seminorm for $H_{0}^{1}(\Omega)$ as

$$
\begin{gathered}
\|\boldsymbol{\tau}\|_{a}^{2}:=a(\boldsymbol{\tau}, \boldsymbol{\tau}), \\
\|v\|_{\mathcal{c}}^{2}:=c(v, v) \quad \forall \boldsymbol{\tau} \in \mathbf{L}^{2}(\Omega, \mathbb{S}), v \in H_{0}^{1}(\Omega) .
\end{gathered}
$$

Throughout this paper, we also use " $\lesssim \ldots$. to mean that " $\leq C$...", where $C$ is a generic positive constant independent of $h$, which may take different values at different appearances. And $A \approx B$ means $A \lesssim B$ and $B \lesssim A$.

Let $\mathbf{R}_{h}: \mathbf{L}^{2}(\Omega, \mathbb{S}) \rightarrow \boldsymbol{\Sigma}_{h} \cap \mathbf{H}^{1}(\Omega, \mathbb{S})$ be the Clément interpolation operator introduced in [30]. Then we define an interpolation operator $\Pi_{h}: \Sigma \rightarrow \Sigma_{h}$ based on the degrees 
of freedom of Arnold-Winther element space as follows (cf. [11]): for any $\boldsymbol{\tau} \in \Sigma, K \in \mathscr{T}_{h}$,

$$
\begin{gathered}
\Pi_{h} \boldsymbol{\tau}(p)=\mathbf{R}_{h} \boldsymbol{\tau}(p) \quad \text { for all vertices } p \text { of } K, \\
\int_{e}\left(\boldsymbol{\tau}-\boldsymbol{\Pi}_{h} \boldsymbol{\tau}\right) \mathbf{n} \cdot \mathbf{v} d s=0
\end{gathered}
$$$$
\text { for all edges } e \text { of } K \text { and } \mathbf{v} \in\left(P_{k-2}(e)\right)^{2} \text {, }
$$$$
\int_{K}\left(\boldsymbol{\tau}-\boldsymbol{\Pi}_{h} \tau\right): \boldsymbol{\varsigma} d x=0 \quad \forall \boldsymbol{\varsigma} \in \mathbf{N}_{k-2}(K) .
$$

For any $\boldsymbol{\tau} \in \Sigma$, by the definition of $\Pi_{h}$, it holds that (cf. [11])

$$
\int_{\Omega}\left(\boldsymbol{\nabla} \cdot\left(\boldsymbol{\tau}-\boldsymbol{\Pi}_{h} \boldsymbol{\tau}\right)\right) \cdot \mathbf{v} d x=0 \quad \forall \mathbf{v} \in \mathbf{X}_{k-2}
$$

Then we define the second interpolation operator $I_{h}: V \cap$ $H^{2}(\Omega) \rightarrow V_{h}$ in the following way (cf. $[4,5]$ ): given $w \in$ $V \cap H^{2}(\Omega)$, for any element $K \in \mathscr{T}_{h}$, any vertex $a$ of $K$, and any edge $e$ of $K, I_{h} w \in V_{h}$ satisfies

$$
\begin{gathered}
I_{h} w(a)=w(a), \\
\int_{e}\left(w-I_{h} w\right) v d s=0 \quad \forall v \in P_{k-2}(e), \\
\int_{K}\left(w-I_{h} w\right) v d x=0 \quad \forall v \in P_{k-3}(K) .
\end{gathered}
$$

According to Proposition 5.1 in [31] and (11), we have for all $w \in V \cap H^{2}(\Omega)$,

$$
b\left(\boldsymbol{\tau}, w-I_{h} w\right)=0 \quad \forall \boldsymbol{\tau} \in \boldsymbol{\Sigma}_{h} .
$$

For simplicity, we still write $I_{h}, \mathbf{Q}_{k-2}$ and $\Pi_{h}$ for $\left.I_{h}\right|_{K}$, $\left.\mathbf{Q}_{k-2}\right|_{K}$ and $\left.\boldsymbol{\Pi}_{h}\right|_{K}$. The error estimates for interpolation operators $I_{h}, \mathbf{Q}_{k-2}$, and $\boldsymbol{\Pi}_{h}$ are summarized in the following lemma (cf. $[1,4,5,11,20,31])$.

Lemma 2. For all $v \in H^{m+2}(K), \boldsymbol{\tau} \in \mathbf{H}^{m}(\Omega, \mathbb{S})$ with $m a$ nonnegative integer, and all $K \in \mathscr{T}_{h}$, one has the estimates

$$
\begin{aligned}
& \left\|v-I_{h} v\right\|_{0, K}+h_{K}^{1 / 2}\left\|v-I_{h} v\right\|_{0, \partial K}+h_{K}\left|v-I_{h} v\right|_{1, K} \\
& \quad \leq h_{K}^{\min \{m+1, k\}+1}\|v\|_{m+2, K}, \\
& \left\|\nabla v-\mathbf{Q}_{k-2}(\nabla v)\right\|_{0, K} \lesssim h_{K}^{\min \{m+1, k-1\}}\|v\|_{m+2, K}, \\
& \left\|\boldsymbol{\tau}-\boldsymbol{\Pi}_{h} \boldsymbol{\tau}\right\|_{0}+h\left\|\nabla \cdot\left(\boldsymbol{\tau}-\boldsymbol{\Pi}_{h} \boldsymbol{\tau}\right)\right\|_{0} \lesssim h^{\min \{m, k\}}\|\boldsymbol{\tau}\|_{m} .
\end{aligned}
$$

For any $v \in H^{m+2}(\Omega)$ with $m$ a nonnegative integer, we obtain from Lemma 2 and triangle inequality

$$
\begin{aligned}
\| \nabla & \left(I_{h} v\right)-\mathbf{Q}_{k-2}\left(\nabla\left(I_{h} v\right)\right) \|_{0, K} \\
\leq & \left\|\nabla\left(v-I_{h} v\right)\right\|_{0, K}+\left\|\mathbf{Q}_{k-2}\left(\nabla\left(v-I_{h} v\right)\right)\right\|_{0, K} \\
& +\left\|\nabla v-\mathbf{Q}_{k-2}(\nabla v)\right\|_{0, K}
\end{aligned}
$$

$$
\begin{aligned}
& \leq 2\left\|\nabla\left(v-I_{h} v\right)\right\|_{0, K}+\left\|\nabla v-\mathbf{Q}_{k-2}(\nabla v)\right\|_{0, K} \\
& \leq h_{K}^{\min \{m+1, k-1\}}|v|_{m+2, K},
\end{aligned}
$$$$
\left\|I_{h} v\right\|_{c}^{2}=\sum_{K \in \mathscr{T}_{h}} h_{K}^{-2 \alpha} \| \nabla\left(I_{h} v\right)
$$$$
-\mathbf{Q}_{k-2}\left(\nabla\left(I_{h} v\right)\right) \|_{0, K}^{2}
$$$$
\lesssim h^{2 \min \{m+1, k-1\}-2 \alpha}\|v\|_{m+2}^{2} \text {. }
$$

3.2. A Priori Error Analysis. Subtracting (12)-(13) from (4)(5), we obtain the following error equations:

$$
\begin{gathered}
a\left(\boldsymbol{\sigma}-\boldsymbol{\sigma}_{h}, \boldsymbol{\tau}\right)+b\left(\boldsymbol{\tau}, u-u_{h}\right)=0 \quad \forall \boldsymbol{\tau} \in \boldsymbol{\Sigma}_{h}, \\
b\left(\boldsymbol{\sigma}-\boldsymbol{\sigma}_{h}, v\right)+c\left(u_{h}, v\right)=0 \quad \forall v \in V_{h} .
\end{gathered}
$$

Using (21), the last error equations can be rewritten as

$$
\begin{gathered}
a\left(\boldsymbol{\sigma}-\boldsymbol{\sigma}_{h}, \boldsymbol{\tau}\right)+b\left(\boldsymbol{\tau}, I_{h} u-u_{h}\right)=0 \quad \forall \boldsymbol{\tau} \in \boldsymbol{\Sigma}_{h}, \\
b\left(\boldsymbol{\Pi}_{h} \boldsymbol{\sigma}-\boldsymbol{\sigma}_{h}, v\right)+c\left(u_{h}, v\right)=b\left(\boldsymbol{\Pi}_{h} \boldsymbol{\sigma}-\boldsymbol{\sigma}, v\right) \quad \forall v \in V_{h} .
\end{gathered}
$$

Theorem 3. Assume that the solution $(\sigma, u)$ for mixed formulation (4)-(5) satisfies $\boldsymbol{\sigma} \in \mathbf{H}^{m}(\Omega, \mathbb{S})$ and $u \in H^{m+2}(\Omega)$ for some positive integer $m$, and let $\left(\sigma_{h}, u_{h}\right)$ be the solution of stabilized mixed finite element methods (12)-(13). Then

$$
\left\|\boldsymbol{\sigma}-\boldsymbol{\sigma}_{h}\right\|_{a}+\left\|u_{h}\right\|_{c} \lesssim h^{\min \{m-1+\alpha, k-1-\alpha\}}\left(\|\boldsymbol{\sigma}\|_{m}+\|u\|_{m+2}\right) .
$$

Proof. Choosing $\boldsymbol{\tau}=\boldsymbol{\Pi}_{h} \boldsymbol{\sigma}-\boldsymbol{\sigma}_{h}$ in (26) and $v=I_{h} u-u_{h}$ in (27), and subtracting (27) from (26), we get

$$
\begin{gathered}
a\left(\boldsymbol{\sigma}-\boldsymbol{\sigma}_{h}, \boldsymbol{\Pi}_{h} \boldsymbol{\sigma}-\boldsymbol{\sigma}_{h}\right)-c\left(u_{h}, I_{h} u-u_{h}\right) \\
=b\left(\boldsymbol{\sigma}-\boldsymbol{\Pi}_{h} \boldsymbol{\sigma}, I_{h} u-u_{h}\right) .
\end{gathered}
$$

Then we have from (19)

$$
\begin{aligned}
a\left(\boldsymbol{\Pi}_{h} \boldsymbol{\sigma}-\boldsymbol{\sigma}_{h}, \boldsymbol{\Pi}_{h} \boldsymbol{\sigma}-\boldsymbol{\sigma}_{h}\right)+c\left(u_{h}, u_{h}\right) \\
=a\left(\boldsymbol{\Pi}_{h} \boldsymbol{\sigma}-\boldsymbol{\sigma}, \boldsymbol{\Pi}_{h} \boldsymbol{\sigma}-\boldsymbol{\sigma}_{h}\right) \\
+a\left(\boldsymbol{\sigma}-\boldsymbol{\sigma}_{h}, \boldsymbol{\Pi}_{h} \boldsymbol{\sigma}-\boldsymbol{\sigma}_{h}\right)+c\left(u_{h}, u_{h}\right) \\
=a\left(\boldsymbol{\Pi}_{h} \boldsymbol{\sigma}-\boldsymbol{\sigma}, \boldsymbol{\Pi}_{h} \boldsymbol{\sigma}-\boldsymbol{\sigma}_{h}\right) \\
\quad+b\left(\boldsymbol{\sigma}-\boldsymbol{\Pi}_{h} \boldsymbol{\sigma}, I_{h} u-u_{h}\right)+c\left(u_{h}, I_{h} u\right) \\
=a\left(\boldsymbol{\Pi}_{h} \boldsymbol{\sigma}-\boldsymbol{\sigma}, \boldsymbol{\Pi}_{h} \boldsymbol{\sigma}-\boldsymbol{\sigma}_{h}\right)+c\left(u_{h}, I_{h} u\right) \\
\quad-\int_{\Omega}\left(\nabla \cdot\left(\boldsymbol{\sigma}-\boldsymbol{\Pi}_{h} \boldsymbol{\sigma}\right)\right) \\
\quad \cdot\left(\nabla\left(I_{h} u-u_{h}\right)-\mathbf{Q}_{k-2}\left(\nabla\left(I_{h} u-u_{h}\right)\right)\right) d x .
\end{aligned}
$$


Using Cauchy-Schwarz inequality and triangle inequality,

$$
\begin{aligned}
\left\|\boldsymbol{\Pi}_{h} \boldsymbol{\sigma}-\boldsymbol{\sigma}_{h}\right\|_{a}^{2}+\left\|u_{h}\right\|_{c}^{2} \\
\leq\left\|\boldsymbol{\Pi}_{h} \boldsymbol{\sigma}-\boldsymbol{\sigma}\right\|_{a}\left\|\boldsymbol{\Pi}_{h} \boldsymbol{\sigma}-\boldsymbol{\sigma}_{h}\right\|_{a}+\left\|u_{h}\right\|_{c}\left\|I_{h} u\right\|_{c} \\
\quad+h^{\alpha}\left\|\nabla \cdot\left(\boldsymbol{\sigma}-\boldsymbol{\Pi}_{h} \boldsymbol{\sigma}\right)\right\|_{0}\left\|I_{h} u-u_{h}\right\|_{c} \\
\leq\left\|\boldsymbol{\Pi}_{h} \boldsymbol{\sigma}-\boldsymbol{\sigma}\right\|_{a}\left\|\boldsymbol{\Pi}_{h} \boldsymbol{\sigma}-\boldsymbol{\sigma}_{h}\right\|_{a}+\left\|u_{h}\right\|_{c}\left\|I_{h} u\right\|_{c} \\
\quad+h^{\alpha}\left\|\nabla \cdot\left(\boldsymbol{\sigma}-\boldsymbol{\Pi}_{h} \boldsymbol{\sigma}\right)\right\|_{0}\left(\left\|I_{h} u\right\|_{c}+\left\|u_{h}\right\|_{c}\right) .
\end{aligned}
$$

Thus

$$
\begin{aligned}
\| \boldsymbol{\Pi}_{h} \boldsymbol{\sigma} & -\boldsymbol{\sigma}_{h}\left\|_{a}^{2}+\right\| u_{h}\left\|_{c}^{2} \leq\right\| \boldsymbol{\Pi}_{h} \boldsymbol{\sigma}-\boldsymbol{\sigma} \|_{a}^{2} \\
& +2\left\|I_{h} u\right\|_{c}^{2}+2 h^{2 \alpha}\left\|\nabla \cdot\left(\boldsymbol{\sigma}-\boldsymbol{\Pi}_{h} \boldsymbol{\sigma}\right)\right\|_{0}^{2},
\end{aligned}
$$

which together with triangle inequality, Lemma 2, and (24) ends the proof.

Using the usual duality argument, we can additionally derive error estimate of $u_{h}$ in the $H^{1}(\Omega)$-norm. To this end, we assume that $\Omega$ is a convex bounded polygonal domain hereafter in the section. Let $(\widetilde{\boldsymbol{\sigma}}, \widetilde{u})$ be the solution of the auxiliary problem:

$$
\begin{gathered}
a(\boldsymbol{\tau}, \tilde{\boldsymbol{\sigma}})+b(\boldsymbol{\tau}, \tilde{u})=0 \quad \forall \boldsymbol{\tau} \in \boldsymbol{\Sigma}, \\
-b(\tilde{\boldsymbol{\sigma}}, v)=\int_{\Omega} \nabla\left(I_{h} u-u_{h}\right) \cdot \nabla v d x \quad \forall v \in V .
\end{gathered}
$$

Since $\Omega$ is convex, we know $\widetilde{u} \in H^{3}(\Omega) \cap H_{0}^{2}(\Omega)$ with the bound (cf. $[32,33])$

$$
\|\widetilde{\boldsymbol{\sigma}}\|_{1}+\|\widetilde{u}\|_{3} \lesssim\left|I_{h} u-u_{h}\right|_{1} .
$$

Denote by $\left(\widetilde{\boldsymbol{\sigma}}_{h}, \widetilde{u}_{h}\right)$ the corresponding stabilized mixed finite element solution:

$$
\begin{gathered}
a\left(\tilde{\boldsymbol{\sigma}}_{h}, \boldsymbol{\tau}\right)+b\left(\boldsymbol{\tau}, \tilde{u}_{h}\right)=0 \quad \forall \boldsymbol{\tau} \in \Sigma_{h}, \\
-b\left(\tilde{\boldsymbol{\sigma}}_{h}, v\right)+c\left(\tilde{u}_{h}, v\right)=\int_{\Omega} \nabla\left(I_{h} u-u_{h}\right) \cdot \nabla v d x \quad \forall v \in V_{h} .
\end{gathered}
$$

As (26)-(27), we have the following error equations for auxiliary problem:

$$
\begin{gathered}
a\left(\tilde{\boldsymbol{\sigma}}-\tilde{\boldsymbol{\sigma}}_{h}, \boldsymbol{\tau}\right)+b\left(\boldsymbol{\tau}, I_{h} \tilde{u}-\tilde{u}_{h}\right)=0 \quad \forall \boldsymbol{\tau} \in \Sigma_{h}, \\
b\left(\boldsymbol{\Pi}_{h} \tilde{\boldsymbol{\sigma}}-\tilde{\boldsymbol{\sigma}}_{h}, v\right)+c\left(\widetilde{u}_{h}, v\right)=b\left(\boldsymbol{\Pi}_{h} \tilde{\boldsymbol{\sigma}}-\tilde{\boldsymbol{\sigma}}, v\right) \quad \forall v \in V_{h} .
\end{gathered}
$$

By Theorem 3 and regularity (35), we also have

$$
\left\|\widetilde{\boldsymbol{\sigma}}-\widetilde{\boldsymbol{\sigma}}_{h}\right\|_{a}+\left\|\widetilde{u}_{h}\right\|_{c} \lesssim h^{\alpha}\left(\|\widetilde{\boldsymbol{\sigma}}\|_{1}+\|\widetilde{u}\|_{3}\right) \lesssim h^{\alpha}\left|I_{h} u-u_{h}\right|_{1} .
$$

Lemma 4. Let $\Omega$ be a convex bounded polygonal domain. Assume that the solution $(\sigma, u)$ for mixed formulation (4)-(5) satisfies $\boldsymbol{\sigma} \in \mathbf{H}^{m}(\Omega, \mathbb{S})$ and $u \in H^{m+2}(\Omega)$ for some positive integer $m$; then

$$
\begin{aligned}
& b\left(\boldsymbol{\Pi}_{h} \tilde{\boldsymbol{\sigma}}-\tilde{\boldsymbol{\sigma}}, I_{h} u-u_{h}\right) \\
& \quad \lesssim h^{\min \{m-1+2 \alpha, k-1\}}\left(\|\boldsymbol{\sigma}\|_{m}+\|u\|_{m+2}\right)\left|I_{h} u-u_{h}\right|_{1} .
\end{aligned}
$$

Proof. Taking $v=I_{h} u-u_{h}$ in (37), it holds that

$$
\begin{aligned}
& \quad b\left(\Pi_{h} \tilde{\boldsymbol{\sigma}}-\tilde{\boldsymbol{\sigma}}, I_{h} u-u_{h}\right) \\
& \quad=b\left(\Pi_{h} \tilde{\boldsymbol{\sigma}}-\tilde{\boldsymbol{\sigma}}_{h}, I_{h} u-u_{h}\right)+c\left(\tilde{u}_{h}, I_{h} u-u_{h}\right) .
\end{aligned}
$$

On the other side, choosing $\boldsymbol{\tau}=\Pi_{h} \widetilde{\boldsymbol{\sigma}}-\widetilde{\boldsymbol{\sigma}}_{h}$ in (26), we get

$$
b\left(\Pi_{h} \tilde{\boldsymbol{\sigma}}-\tilde{\boldsymbol{\sigma}}_{h}, I_{h} u-u_{h}\right)=-a\left(\boldsymbol{\sigma}-\boldsymbol{\sigma}_{h}, \Pi_{h} \tilde{\boldsymbol{\sigma}}-\widetilde{\boldsymbol{\sigma}}_{h}\right) .
$$

Then it follows from the last two equalities that

$$
\begin{aligned}
b & \left(\boldsymbol{\Pi}_{h} \tilde{\boldsymbol{\sigma}}-\tilde{\boldsymbol{\sigma}}, I_{h} u-u_{h}\right) \\
& =-a\left(\boldsymbol{\sigma}-\boldsymbol{\sigma}_{h}, \Pi_{h} \tilde{\boldsymbol{\sigma}}-\tilde{\boldsymbol{\sigma}}_{h}\right)+c\left(\tilde{u}_{h}, I_{h} u-u_{h}\right) .
\end{aligned}
$$

According to Cauchy-Swarchz inequality, triangle inequality, Lemma 2, (38), and regularity (35),

$$
\begin{aligned}
b\left(\boldsymbol{\Pi}_{h} \tilde{\boldsymbol{\sigma}}-\tilde{\boldsymbol{\sigma}}, I_{h} u-u_{h}\right) \\
\leq\left\|\boldsymbol{\sigma}-\boldsymbol{\sigma}_{h}\right\|_{a}\left\|\boldsymbol{\Pi}_{h} \tilde{\boldsymbol{\sigma}}-\tilde{\boldsymbol{\sigma}}_{h}\right\|_{a}+\left\|\tilde{u}_{h}\right\|_{c}\left\|I_{h} u-u_{h}\right\|_{c} \\
\leq\left\|\boldsymbol{\sigma}-\boldsymbol{\sigma}_{h}\right\|_{a}\left(\left\|\boldsymbol{\Pi}_{h} \tilde{\boldsymbol{\sigma}}-\tilde{\boldsymbol{\sigma}}\right\|_{a}+\left\|\tilde{\boldsymbol{\sigma}}-\tilde{\boldsymbol{\sigma}}_{h}\right\|_{a}\right) \\
\quad+\left\|\tilde{u}_{h}\right\|_{c}\left(\left\|I_{h} u\right\|_{c}+\left\|u_{h}\right\|_{c}\right) \\
\leq h^{\alpha}\left\|\boldsymbol{\sigma}-\boldsymbol{\sigma}_{h}\right\|_{a}\left(\|\tilde{\boldsymbol{\sigma}}\|_{1}+\left|I_{h} u-u_{h}\right|_{1}\right) \\
\quad+h^{\alpha}\left|I_{h} u-u_{h}\right|_{1}\left(\left\|I_{h} u\right\|_{c}+\left\|u_{h}\right\|_{c}\right) \\
\leq h^{\alpha}\left|I_{h} u-u_{h}\right|_{1}\left(\left\|\boldsymbol{\sigma}-\boldsymbol{\sigma}_{h}\right\|_{a}+\left\|u_{h}\right\|_{c}+\left\|I_{h} u\right\|_{c}\right) .
\end{aligned}
$$

Therefore, the proof is finished from last inequality, Theorem 3, and (24).

Theorem 5. Let $\Omega$ be a convex bounded polygonal domain. Assume that the solution $(\sigma, u)$ for mixed formulation (4)-(5) satisfies $\boldsymbol{\sigma} \in \mathbf{H}^{m}(\Omega, \mathbb{S})$ and $u \in H^{m+2}(\Omega)$ for some positive integer $m$, and let $\left(\sigma_{h}, u_{h}\right)$ be the solution of stabilized mixed finite element methods (12)-(13). Then

$$
\left|u-u_{h}\right|_{1} \lesssim h^{\min \{m-1+2 \alpha, k-1\}}\left(\|\boldsymbol{\sigma}\|_{m}+\|u\|_{m+2}\right) .
$$

Proof. Taking $v=I_{h} u-u_{h}$ in (34), we have from Lemma 4

$$
\begin{aligned}
\left|I_{h} u-u_{h}\right|_{1}^{2}= & -b\left(\widetilde{\boldsymbol{\sigma}}, I_{h} u-u_{h}\right) \\
= & -b\left(\widetilde{\boldsymbol{\sigma}}-\boldsymbol{\Pi}_{h} \widetilde{\boldsymbol{\sigma}}, I_{h} u-u_{h}\right)-b\left(\Pi_{h} \tilde{\boldsymbol{\sigma}}, I_{h} u-u_{h}\right) \\
\lesssim & h^{\min \{m-1+2 \alpha, k-1\}}\left(\|\boldsymbol{\sigma}\|_{m}+\|u\|_{m+2}\right)\left|I_{h} u-u_{h}\right|_{1} \\
& -b\left(\boldsymbol{\Pi}_{h} \widetilde{\boldsymbol{\sigma}}, I_{h} u-u_{h}\right) .
\end{aligned}
$$

Choosing $\boldsymbol{\tau}=\Pi_{h} \widetilde{\boldsymbol{\sigma}}$ in error equation (26), it holds that

$$
\begin{aligned}
-b\left(\Pi_{h} \tilde{\boldsymbol{\sigma}}, I_{h} u-u_{h}\right) & =a\left(\boldsymbol{\sigma}-\boldsymbol{\sigma}_{h}, \Pi_{h} \tilde{\boldsymbol{\sigma}}\right) \\
& =a\left(\boldsymbol{\sigma}-\boldsymbol{\sigma}_{h}, \Pi_{h} \tilde{\boldsymbol{\sigma}}-\tilde{\boldsymbol{\sigma}}\right)+a\left(\boldsymbol{\sigma}-\boldsymbol{\sigma}_{h}, \tilde{\boldsymbol{\sigma}}\right) .
\end{aligned}
$$


Picking $\boldsymbol{\tau}=\boldsymbol{\sigma}-\boldsymbol{\sigma}_{h}$ in (33) and using (21) and error equation (25),

$$
\begin{aligned}
a\left(\boldsymbol{\sigma}-\boldsymbol{\sigma}_{h}, \tilde{\boldsymbol{\sigma}}\right) & =-b\left(\boldsymbol{\sigma}-\boldsymbol{\sigma}_{h}, \widetilde{u}\right) \\
& =-b\left(\boldsymbol{\sigma}-\boldsymbol{\sigma}_{h}, \tilde{u}-I_{h} \widetilde{u}\right)-b\left(\boldsymbol{\sigma}-\boldsymbol{\sigma}_{h}, I_{h} \widetilde{u}\right) \\
& =-b\left(\boldsymbol{\sigma}-\boldsymbol{\Pi}_{h} \boldsymbol{\sigma}, \tilde{u}-I_{h} \widetilde{u}\right)+c\left(u_{h}, I_{h} \widetilde{u}\right) .
\end{aligned}
$$

From the last two equalities and Cauchy-Swarchz inequality,

$$
\begin{aligned}
-b & \left(\boldsymbol{\Pi}_{h} \tilde{\boldsymbol{\sigma}}, I_{h} u-u_{h}\right) \\
\leq & \left\|\boldsymbol{\sigma}-\boldsymbol{\sigma}_{h}\right\|_{a}\left\|\boldsymbol{\Pi}_{h} \tilde{\boldsymbol{\sigma}}-\tilde{\boldsymbol{\sigma}}\right\|_{a} \\
& +\left\|\boldsymbol{\nabla} \cdot\left(\boldsymbol{\sigma}-\boldsymbol{\Pi}_{h} \boldsymbol{\sigma}\right)\right\|_{0}\left\|\nabla\left(\widetilde{u}-I_{h} \tilde{u}\right)\right\|_{0}+\left\|u_{h}\right\|_{c}\left\|I_{h} \tilde{u}\right\|_{c} \cdot
\end{aligned}
$$

By (24), Lemma 2, Theorem 3, and regularity (35),

$$
\begin{aligned}
-b\left(\boldsymbol{\Pi}_{h} \tilde{\boldsymbol{\sigma}}, I_{h} u-u_{h}\right) & \\
\lesssim & h^{\min \{m+\alpha, k-\alpha\}}\|\widetilde{\boldsymbol{\sigma}}\|_{1}\left(\|\boldsymbol{\sigma}\|_{m}+\|u\|_{m+2}\right) \\
& +h^{\min \{m+1, k+1\}}\|\widetilde{u}\|_{3}\|\boldsymbol{\sigma}\|_{m} \\
& +h^{\min \{m+1, k+1-2 \alpha\}}\|\widetilde{u}\|_{3}\left(\|\boldsymbol{\sigma}\|_{m}+\|u\|_{m+2}\right) \\
\lesssim & h^{\min \{m+\alpha, k-\alpha\}}\left(\|\widetilde{\boldsymbol{\sigma}}\|_{1}+\|\widetilde{u}\|_{3}\right)\left(\|\boldsymbol{\sigma}\|_{m}+\|u\|_{m+2}\right) \\
\lesssim & h^{\min \{m+\alpha, k-\alpha\}}\left(\|\boldsymbol{\sigma}\|_{m}+\|u\|_{m+2}\right)\left|I_{h} u-u_{h}\right|_{1},
\end{aligned}
$$

which together with (45) gives

$$
\left|I_{h} u-u_{h}\right|_{1} \lesssim h^{\min \{m-1+2 \alpha, k-1\}}\left(\|\sigma\|_{m}+\|u\|_{m+2}\right) .
$$

Combined with triangle inequality and Lemma 2, we finish the proof.

\section{A Posteriori Error Analysis}

In this section, we intend to investigate the a posteriori error estimates of stabilized mixed finite element methods (12)(13).

4.1. Preliminaries. For any interior edge $e \in \mathscr{E}_{h}^{i}$, let $K^{+}$and $K^{-}$be the two adjacent triangles sharing edge $e$. Denote by $\mathbf{n}^{+}$and $\mathbf{n}^{-}$the unit outward normals to the common edge $e$ of the triangles $K^{+}$and $K^{-}$, respectively. For any vector-valued function $\phi$, write $\phi^{+}=\left.\phi\right|_{K^{+}}$and $\phi^{-}=\left.\phi\right|_{K^{-}}$. Then define jump on $e$ as follows:

$$
[\phi]=\phi^{+} \cdot \mathbf{n}^{+}+\phi^{-} \cdot \mathbf{n}^{-} .
$$

If an edge $e$ lies on the boundary $\partial \Omega$, jump is defined by

$$
[\phi]=\phi \cdot \mathbf{n} .
$$

Based on the triangulation $\mathscr{T}_{h}$, let

$$
W:=\left\{v \in H_{0}^{1}(\Omega):\left.v\right|_{K} \in H^{2}(K) \forall K \in \mathscr{T}_{h}\right\} .
$$

And equip $W$ with a broken energy norm as follows:

$$
\|v\|^{2}:=|v|_{2, h}^{2}+\sum_{e \in \mathscr{E}_{h}} h_{e}^{-1}\|[\nabla v]\|_{0, e}^{2} \quad \forall v \in W .
$$

Define a seminorm for $H_{0}^{1}(\Omega)$ as

$$
\left.\|v\|_{\widetilde{\mathcal{c}}}^{2}:=\sum_{K \in \mathscr{T}_{h}} h_{K}^{2-4 \alpha} \| \nabla v-\mathbf{Q}_{k-2}(\nabla v)\right) \|_{0, K}^{2} \quad \forall v \in H_{0}^{1}(\Omega) .
$$

For any $\boldsymbol{\tau} \in \boldsymbol{\Sigma}_{h}$ and $v \in V_{h}$, define error estimator as

$$
\begin{aligned}
\eta_{h}^{2}(\boldsymbol{\tau}, v, f) & :=\sum_{K \in \mathscr{T}_{h}}\left(h_{K}^{4}\|f+\nabla \cdot(\nabla \cdot \boldsymbol{\tau})\|_{0, K}^{2}+\|\boldsymbol{\tau}-\mathscr{C}(\mathscr{K}(v))\|_{0, K}^{2}\right) \\
& +\sum_{e \in \mathscr{C}_{h}^{i}} h_{e}^{3}\|[\nabla \cdot \boldsymbol{\tau}]\|_{0, e}^{2}+\sum_{e \in \mathscr{E}_{h}} h_{e}^{-1}\|[\nabla v]\|_{0, e}^{2}+\|v\|_{\tilde{c}}^{2} .
\end{aligned}
$$

And the oscillation is defined as

$$
\operatorname{osc}_{h}^{2}(f):=\sum_{K \in \mathscr{T}_{h}} h_{K}^{4}\left\|f-Q_{l} f\right\|_{0, K}^{2} .
$$

For any vertex $p$ and edge $e$ of triangulation $\mathscr{T}_{h}$, denote $\mathscr{T}_{p}$ and $\mathscr{T}_{e}$ by the set of triangles in $\mathscr{T}_{h}$ sharing common vertex $p$ and edge $e$, respectively. For any subset $\mathcal{S}$ of $\mathscr{T}_{h}$, let $|\mathcal{S}|$ be the cardinalities of $\mathcal{S}$. For any edge $e$ of triangulation $\mathscr{T}_{h}, \omega_{e}$ means the union of elements in $\mathscr{T}_{e}$.

To show the reliability of the error estimator introduced previously, we need a $H_{0}^{2}(\Omega)$-conforming finite element space and corresponding connection operator. Here we intend to use the Zienkiewicz-Guzmán-Neilan finite element space associated with $\mathscr{T}_{h}$ (cf. $[1,27]$ ), by reason that the degrees of freedom only involve the values and integrations of function and first-order derivatives, no any higher-order derivatives. For any $K \in \mathscr{T}_{h}$, denote by $\left\{p_{i}\right\}_{i=1}^{3},\left\{e_{i}\right\}_{i=1}^{3}$ the three vertices and edges of $K$ and $\left\{\lambda_{i}\right\}_{i=1}^{3}$ the three corresponding barycentric coordinates such that $\lambda_{i}\left(p_{j}\right)=\delta_{i j}$. Define the triangle-bubble function and edge-bubble functions as

$$
b_{K}:=\lambda_{1} \lambda_{2} \lambda_{3}, \quad b_{i}:=\lambda_{i+1} \lambda_{i+2}
$$

for $i=1,2,3$, where $i+1$ and $i+2$ are taken as values modulo 3 , respectively. Moreover, define rational edge bubble functions as $(i=1,2,3)$

$$
B_{i}:= \begin{cases}\frac{b_{K} b_{i}}{\left(\lambda_{i}+\lambda_{i+1}\right)\left(\lambda_{i}+\lambda_{i+2}\right)} & \text { for } 0 \leq \lambda_{i} \leq 1,0 \leq \lambda_{i+1}, \lambda_{i+2}<1, \\ 0 & \text { otherwise. }\end{cases}
$$

Then the local and global Zienkiewicz-Guzmán-Neilan finite element spaces given in [27] are

$$
\begin{gathered}
U_{K}:=P_{k}(K)+\operatorname{span}\left\{b_{i} \lambda_{i+1}^{k-1}\right\}_{i=1}^{3}+\operatorname{span}\left\{B_{i} Q_{k-2}^{(i)}(K)\right\}_{i=1}^{3}, \\
U_{h}:=\left\{w \in H_{0}^{2}(\Omega):\left.w\right|_{K} \in U_{K}\right\},
\end{gathered}
$$


where

$$
\begin{aligned}
& Q_{k-2}^{(i)}(K) \\
& \quad:=\left\{w \in P_{k-2}(K): \int_{K} B_{i} w v d x=0 \quad \forall v \in P_{k-3}(K)\right\} .
\end{aligned}
$$

In the case $k=2, Q_{k-2}^{(i)}(K)$ is understood as $P_{0}(K)$. The local degrees of freedom are taken as

$$
\begin{gathered}
w\left(p_{i}\right), \nabla w\left(p_{i}\right) \quad \forall \text { vertices } p_{i}, \\
\int_{e_{i}} w v d s \quad \forall v \in P_{k-3}\left(e_{i}\right), \\
\int_{K} w v d x \quad \forall v \in P_{k-3}(K), \\
\int_{e_{i}} \partial_{n} w v d s \quad \forall v \in P_{k-2}\left(e_{i}\right) .
\end{gathered}
$$

Now we can construct a connection operator $L_{h}: V_{h} \rightarrow$ $U_{h}$ by averaging (for details see $[6,34]$ ) in the following way: Given $w \in V_{h}$, for every interior vertex $p$, interior edge $e$, and triangle $K$ of triangulation $\mathscr{T}_{h}$,

$$
\begin{gathered}
L_{h} w(p)=w(p), \quad \nabla L_{h} w(p)=\frac{1}{\left|\mathscr{T}_{p}\right|} \sum_{K^{\prime} \in \mathscr{T}_{p}} \nabla\left(\left.w\right|_{K^{\prime}}\right)(p), \\
\int_{e}\left(w-L_{h} w\right) v d s=0 \quad \forall v \in P_{k-3}(e), \\
\int_{K}\left(w-L_{h} w\right) v d x=0 \quad \forall v \in P_{k-3}(K), \\
\int_{e}\left(\frac{1}{\left|\mathscr{T}_{e}\right|} \sum_{K^{\prime} \in \mathscr{T}_{e}} \partial_{n}\left(\left.w\right|_{K^{\prime}}\right)-\partial_{n}\left(L_{h} w\right)\right) v d s=0
\end{gathered}
$$$$
\forall v \in P_{k-2}(e),
$$

and for every vertex $p$, edge $e$ on $\partial \Omega$,

$$
\begin{gathered}
L_{h} w(p)=0, \quad \nabla L_{h} w(p)=0, \\
\int_{e}\left(L_{h} w\right) v d s=0 \quad \forall v \in P_{k-3}(e), \\
\int_{e} \partial_{n}\left(L_{h} w\right) v d s=0 \quad \forall v \in P_{k-2}(e) .
\end{gathered}
$$

According to the similar arguments in $[6,34,35]$, we can get the following estimate for connection operator $L_{h}$ :

$$
\left|v-L_{h} v\right|_{2, h}^{2} \lesssim \sum_{e \in \mathscr{E}_{h}} h_{e}^{-1}\|[\nabla v]\|_{0, e}^{2} \quad \forall v \in V_{h} .
$$

4.2. A Posteriori Error Analysis. First, let us consider the reliability of the a posteriori error estimator. We will follow the similar argument as in [6] by replacing Hsieh-Clough-Tocher element space by Zienkiewicz-Guzmán-Neilan element space proposed in [27].
Theorem 6. Let $(\sigma, u)$ and $\left(\sigma_{h}, u_{h}\right)$ be solutions of mixed formulation (4)-(5) and stabilized mixed finite element methods (12)-(13), respectively. Then

$$
\left\|\boldsymbol{\sigma}-\boldsymbol{\sigma}_{h}\right\|_{0}+\left|\left\|u-u_{h}\right\|\right|+\left\|u_{h}\right\|_{\tilde{c}} \lesssim \eta_{h}\left(\sigma_{h}, u_{h}, f\right) .
$$

Proof. Letting $w=u-L_{h} u_{h}$, then $w \in H_{0}^{2}(\Omega)$. Using integration by parts, (5) and (13), we have

$$
\begin{aligned}
\int_{\Omega} & \left(\boldsymbol{\sigma}-\boldsymbol{\sigma}_{h}\right): \mathscr{K}(w) d x \\
= & -b\left(\boldsymbol{\sigma}-\boldsymbol{\sigma}_{h}, w\right) \\
= & \int_{\Omega} f w d x+b\left(\boldsymbol{\sigma}_{h}, w\right) \\
= & \int_{\Omega} f\left(w-I_{h} w\right) d x+b\left(\boldsymbol{\sigma}_{h}, w-I_{h} w\right)+c\left(u_{h}, I_{h} w\right) \\
= & \sum_{K \in \mathscr{T}_{h}} \int_{K}\left(f+\nabla \cdot\left(\nabla \cdot \boldsymbol{\sigma}_{h}\right)\right)\left(w-I_{h} w\right) d x \\
& -\sum_{e \in \mathscr{G}_{h}^{i}} \int_{e}\left[\nabla \cdot \boldsymbol{\sigma}_{h}\right]\left(w-I_{h} w\right) d s+c\left(u_{h}, I_{h} w\right) .
\end{aligned}
$$

Then by Cauchy-Swarchz inequality, Lemma 2, and (23), we get

$$
\begin{aligned}
\int_{\Omega}\left(\boldsymbol{\sigma}-\boldsymbol{\sigma}_{h}\right): \mathscr{K}(w) d x \\
\leq\left(\sum_{K \in \mathscr{T}_{h}} h_{K}^{4}\left\|f+\nabla \cdot\left(\nabla \cdot \boldsymbol{\sigma}_{h}\right)\right\|_{0, K}^{2}\right)^{1 / 2}|w|_{2} \\
\quad+\left(\sum_{e \in \mathscr{G}_{h}^{i}} h_{e}^{3}\left\|\left[\nabla \cdot \boldsymbol{\sigma}_{h}\right]\right\|_{0, e}^{2}\right)^{1 / 2}|w|_{2}+\left\|u_{h}\right\|_{\tilde{c}}|w|_{2} .
\end{aligned}
$$

It follows from Cauchy-Swarchz inequality, triangular inequality, and (67) that

$$
\begin{aligned}
\int_{\Omega} & \left(\sigma_{h}-\mathscr{C}\left(\mathscr{K}\left(L_{h} u_{h}\right)\right)\right): \mathscr{K}(w) d x \\
\leq & \left\|\sigma_{h}-\mathscr{C}\left(\mathscr{K}\left(L_{h} u_{h}\right)\right)\right\|_{0}|w|_{2} \\
\leq & \left.\left\|\sigma_{h}-\mathscr{C}\left(\mathscr{K}_{h}\left(u_{h}\right)\right)\right\|_{0}+\left|u_{h}-L_{h} u_{h}\right|_{2, h}\right)|w|_{2} \\
\leq & \left(\left\|\sigma_{h}-\mathscr{C}\left(\mathscr{K}_{h}\left(u_{h}\right)\right)\right\|_{0}\right. \\
& \left.+\left(\sum_{e \in \mathscr{E}_{h}} h_{e}^{-1}\left\|\left[\nabla u_{h}\right]\right\|_{0, e}^{2}\right)^{1 / 2}\right)|w|_{2},
\end{aligned}
$$


where $\mathscr{K}_{h}$ means the discrete version of $\mathscr{K}$ associated with triangulation $\mathscr{T}_{h}$. Combining the last two inequalities, we obtain by the fact that $\sigma=\mathscr{C}(\mathscr{K}(u))$

$$
\begin{aligned}
\left|u-L_{h} u_{h}\right|_{2}^{2} \leq & \int_{\Omega}\left(\boldsymbol{\sigma}-\boldsymbol{\sigma}_{h}\right): \mathscr{K}(w) d x \\
& +\int_{\Omega}\left(\sigma_{h}-\mathscr{C}\left(\mathscr{K}\left(L_{h} u_{h}\right)\right)\right): \mathscr{K}(w) d x \\
\lesssim & \eta_{h}\left(\sigma_{h}, u_{h}, f\right)\left|u-L_{h} u_{h}\right|_{2} .
\end{aligned}
$$

Thus, we have

$$
\left|u-L_{h} u_{h}\right|_{2} \lesssim \eta_{h}\left(\sigma_{h}, u_{h}, f\right) .
$$

Then triangular inequality and (67) imply

$$
\begin{aligned}
\left|u-u_{h}\right|_{2, h} \\
\quad \leq\left|u-L_{h} u_{h}\right|_{2}+\left|L_{h} u_{h}-u_{h}\right|_{2, h} \\
\quad \leq \eta_{h}\left(\sigma_{h}, u_{h}, f\right)+\left(\sum_{e \in \mathscr{E}_{h}} h_{e}^{-1}\left\|\left[\nabla u_{h}\right]\right\|_{0, e}^{2}\right)^{1 / 2} \\
\quad \leq \eta_{h}\left(\sigma_{h}, u_{h}, f\right) .
\end{aligned}
$$

Together with triangular inequality, it holds that

$$
\begin{aligned}
\left\|\boldsymbol{\sigma}-\boldsymbol{\sigma}_{h}\right\|_{0} & \leq\left\|\boldsymbol{\sigma}-\mathscr{C}\left(\mathscr{K}_{h}\left(u_{h}\right)\right)\right\|_{0}+\left\|\boldsymbol{\sigma}_{h}-\mathscr{C}\left(\mathscr{K}_{h}\left(u_{h}\right)\right)\right\|_{0} \\
& \leqslant\left|u-u_{h}\right|_{2, h}+\left\|\sigma_{h}-\mathscr{C}\left(\mathscr{K}_{h}\left(u_{h}\right)\right)\right\|_{0} \\
& \leqslant \eta_{h}\left(\sigma_{h}, u_{h}, f\right) .
\end{aligned}
$$

Finally, noting the fact $[\nabla u]=0$ on $\mathscr{E}_{h}$, it follows from the last two inequality that

$$
\begin{aligned}
\| \boldsymbol{\sigma} & -\boldsymbol{\sigma}_{h}\left\|_{0}^{2}+\right\| u-u_{h} \|^{2} \\
& =\left\|\boldsymbol{\sigma}-\boldsymbol{\sigma}_{h}\right\|_{0}^{2}+\left|u-u_{h}\right|_{2, h}^{2}+\sum_{e \in \mathscr{E}_{h}} h_{e}^{-1}\left\|\left[\nabla\left(u-u_{h}\right)\right]\right\|_{0, e}^{2} \\
& =\left\|\boldsymbol{\sigma}-\sigma_{h}\right\|_{0}^{2}+\left|u-u_{h}\right|_{2, h}^{2}+\sum_{e \in \mathscr{E}_{h}} h_{e}^{-1}\left\|\left[\nabla u_{h}\right]\right\|_{0, e}^{2} \\
& \leqslant \eta_{h}^{2}\left(\sigma_{h}, u_{h}, f\right),
\end{aligned}
$$

which together with the definition of $\eta_{h}$ ends the proof.

Then, we study the efficiency of the a posteriori error estimator by bubble function argument.

Lemma 7. Let $(\boldsymbol{\sigma}, u)$ and $\left(\boldsymbol{\sigma}_{h}, u_{h}\right)$ be solutions to mixed formulation (4)-(5) and stabilized mixed finite element methods (12)-(13), respectively. Then for all $K \in \mathscr{T}_{h}$,

$$
h_{K}^{2}\left\|f+\nabla \cdot\left(\nabla \cdot \sigma_{h}\right)\right\|_{0, K} \lesssim\left\|\sigma-\sigma_{h}\right\|_{0, K}+h_{K}^{2}\left\|f-Q_{l} f\right\|_{0, K} .
$$

Proof. Let $\psi_{K}:=\left(Q_{l} f+\nabla \cdot\left(\nabla \cdot \sigma_{h}\right)\right) b_{K}^{2}$. It is obvious that $\psi_{K} \in H_{0}^{2}(K)$ and

$$
\left\|\psi_{K}\right\|_{0, K} \approx\left\|Q_{l} f+\nabla \cdot\left(\nabla \cdot \sigma_{h}\right)\right\|_{0, K}
$$

Using integration by parts and (5) with $v=\psi_{K}$, it follows that

$$
\begin{aligned}
\int_{K}(f & \left.+\nabla \cdot\left(\nabla \cdot \boldsymbol{\sigma}_{h}\right)\right) \psi_{K} d x \\
& =\int_{K} f \psi_{K} d x+b\left(\boldsymbol{\sigma}_{h}, \psi_{K}\right) \\
& =b\left(\boldsymbol{\sigma}_{h}-\boldsymbol{\sigma}, \psi_{K}\right)=\int_{K}\left(\boldsymbol{\sigma}-\boldsymbol{\sigma}_{h}\right): \mathscr{K}\left(\psi_{K}\right) d x
\end{aligned}
$$

Together with standard scaling argument, Cauchy-Swarchz inequality, and inverse inequality, we have

$$
\begin{aligned}
\| Q_{l} f & +\nabla \cdot\left(\nabla \cdot \boldsymbol{\sigma}_{h}\right) \|_{0, K}^{2} \\
& \lesssim \int_{K}\left(Q_{l} f+\nabla \cdot\left(\nabla \cdot \boldsymbol{\sigma}_{h}\right)\right) \psi_{K} d x \\
& =\int_{K}\left(f+\nabla \cdot\left(\nabla \cdot \boldsymbol{\sigma}_{h}\right)\right) \psi_{K} d x+\int_{K}\left(Q_{l} f-f\right) \psi_{K} d x \\
& =\int_{K}\left(\boldsymbol{\sigma}-\boldsymbol{\sigma}_{h}\right): \mathscr{K}\left(\psi_{K}\right) d x+\int_{K}\left(Q_{l} f-f\right) \psi_{K} d x \\
& \lesssim\left(h_{K}^{-2}\left\|\boldsymbol{\sigma}-\boldsymbol{\sigma}_{h}\right\|_{0, K}+\left\|f-Q_{l} f\right\|_{0, K}\right)\left\|\psi_{K}\right\|_{0, K},
\end{aligned}
$$

which together with (78) shows

$$
h_{K}^{2}\left\|Q_{l} f+\nabla \cdot\left(\nabla \cdot \sigma_{h}\right)\right\|_{0, K} \lesssim\left\|\boldsymbol{\sigma}-\sigma_{h}\right\|_{0, K}+h_{K}^{2}\left\|f-Q_{l} f\right\|_{0, K} \cdot
$$

Therefore, we can obtain (77) by the last inequality and triangular inequality.

Lemma 8. Let $(\sigma, u)$ and $\left(\sigma_{h}, u_{h}\right)$ be solutions to mixed formulation (4)-(5) and stabilized mixed finite element methods (12)-(13), respectively. Then for all $e \in \mathscr{E}_{h}^{i}$,

$$
h_{e}^{3 / 2}\left\|\left[\nabla \cdot \sigma_{h}\right]\right\|_{0, e} \lesssim\left\|\sigma-\sigma_{h}\right\|_{0, \omega_{e}}+h_{e}^{2}\left\|f-Q_{l} f\right\|_{0, \omega_{e}} .
$$

Proof. Let $K_{1}, K_{2} \in \mathscr{T}_{h}$ such that $e$ is common shared edge of $K_{1}$ and $K_{2}$, and define edge bubble function as (cf. $[19,36]$ )

$$
b_{e}= \begin{cases}\left(\lambda_{K_{1}, 1} \lambda_{K_{1}, 2} \lambda_{K_{2}, 1} \lambda_{K_{2}, 2}\right)^{2} & \text { in } \omega_{e}, \\ 0 & \text { in } \Omega \backslash \omega_{e},\end{cases}
$$

where $\lambda_{K_{1}, i}$ and $\lambda_{K_{2}, i}$ for $i=1,2$ are barycentric coordinates of $K_{1}$ and $K_{2}$ associated with two end points of $e$, respectively. Set $J_{e}:=\left.\left[\nabla \cdot \sigma_{h}\right]\right|_{e} \cdot E_{h}\left(J_{e}\right)$ is defined by extending the jump $J_{e}$ to $\omega_{e}$ constantly along the normal to $e$. Thus $E_{h}\left(J_{e}\right)$ is a piecewise polynomial of degree $k-2$ on $\omega_{e}$ and $\psi_{e}:=$ $b_{e}^{2} E_{h}\left(J_{e}\right) \in H_{0}^{2}\left(\omega_{e}\right)$. It is easy to check that

$$
\left\|\psi_{e}\right\|_{0, \omega_{e}} \lesssim h_{e}^{1 / 2}\left\|J_{e}\right\|_{0, e} .
$$


By standard scaling argument, integration by parts, and (5) with $v=\psi_{e}$, it follows that

$$
\begin{aligned}
& \left\|J_{e}\right\|_{0, e}^{2} \\
& \quad \leq \int_{e} J_{e} \psi_{e} d s \\
& =\sum_{K \in \mathscr{T}_{e}} \int_{K} \psi_{e} \nabla \cdot\left(\nabla \cdot \boldsymbol{\sigma}_{h}\right) d x-b\left(\boldsymbol{\sigma}_{h}, \psi_{e}\right) \\
& =\sum_{K \in \mathscr{T}_{e}} \int_{K}\left(f+\nabla \cdot\left(\nabla \cdot \boldsymbol{\sigma}_{h}\right)\right) \psi_{e} d x+b\left(\boldsymbol{\sigma}-\boldsymbol{\sigma}_{h}, \psi_{e}\right) \\
& =\sum_{K \in \mathscr{T}_{e}} \int_{K}\left(f+\nabla \cdot\left(\nabla \cdot \boldsymbol{\sigma}_{h}\right)\right) \psi_{e} d x \\
& \quad-\sum_{K \in \mathscr{T}_{e}} \int_{K}\left(\boldsymbol{\sigma}-\boldsymbol{\sigma}_{h}\right): \mathscr{K}\left(\psi_{e}\right) d x .
\end{aligned}
$$

Then from Cauchy-Schwarz inequality, (77), inverse inequality, and (84),

$$
\begin{aligned}
& \left\|J_{e}\right\|_{0, e}^{2} \\
& \qquad \sum_{K \in \mathscr{T}_{e}}\left(\left\|f+\nabla \cdot\left(\nabla \cdot \boldsymbol{\sigma}_{h}\right)\right\|_{0, K}\left\|\psi_{e}\right\|_{0, K}\right. \\
& \left.\quad+\left\|\boldsymbol{\sigma}-\boldsymbol{\sigma}_{h}\right\|_{0, K}\left|\psi_{e}\right|_{2, K}\right) \\
& \lesssim \sum_{K \in \mathscr{T}_{e}}\left(h_{K}^{-2}\left\|\boldsymbol{\sigma}-\boldsymbol{\sigma}_{h}\right\|_{0, K}+\left\|f-Q_{l} f\right\|_{0, K}\right)\left\|\psi_{e}\right\|_{0, K} \\
& \lesssim h_{e}^{1 / 2}\left\|J_{e}\right\|_{0, e} \sum_{K \in \mathscr{T}_{e}}\left(h_{K}^{-2}\left\|\boldsymbol{\sigma}-\boldsymbol{\sigma}_{h}\right\|_{0, K}+\left\|f-Q_{l} f\right\|_{0, K}\right) .
\end{aligned}
$$

Therefore, we can conclude (82) by canceling $\left\|J_{e}\right\|_{0, e}$ on both sides and the fact that $h_{e} \approx h_{K}$.

Theorem 9. Let $(\boldsymbol{\sigma}, u)$ and $\left(\boldsymbol{\sigma}_{h}, u_{h}\right)$ be solutions for mixed formulation (4)-(5) and stabilized mixed finite element methods (12)-(13), respectively. Then

$$
\eta_{h}\left(\sigma_{h}, u_{h}, f\right) \lesssim\left\|\sigma-\sigma_{h}\right\|_{0}+\left\|\left|u-u_{h}\right|\right\|+\left\|u_{h}\right\|_{\tilde{c}}+\operatorname{osc}_{h}(f) .
$$

Proof. Noting that $\sigma=\mathscr{C}(\mathscr{K}(u))$, it follows from triangular inequality that

$$
\begin{aligned}
& \| \boldsymbol{\sigma}_{h}-\mathscr{C}\left(\mathscr{K}\left(u_{h}\right)\right) \|_{0, K} \\
& \leq\left\|\boldsymbol{\sigma}-\boldsymbol{\sigma}_{h}\right\|_{0, K}+\left\|\mathscr{C}(\mathscr{K}(u))-\mathscr{C}\left(\mathscr{K}\left(u_{h}\right)\right)\right\|_{0, K} \\
& \quad \leq\left\|\boldsymbol{\sigma}-\boldsymbol{\sigma}_{h}\right\|_{0, K}+\left|u-u_{h}\right|_{2, K} \cdot
\end{aligned}
$$

Hence, the proof is finished by combining the last inequality, (77), (82), and the definition of $\eta_{h}\left(\sigma_{h}, u_{h}, f\right)$ and $\left\|\left|u-u_{h}\right|\right\|$.

\section{Acknowledgments}

This work was partly supported by the NNSFC (Grants nos. 11126226 and 11171257) and Zhejiang Provincial Natural Science Foundation of China (Y6110240, LY12A01015).

\section{References}

[1] P. G. Ciarlet, The Finite Element Method for Elliptic Problems, North-Holland, Amsterdam, The Netherlands, 1978.

[2] P. G. Ciarlet and P.-A. Raviart, "A mixed finite element method for the biharmonic equation," in Mathematical Aspects of Finite Elements in Partial Differential Equations: Proceedings of a Symposium Conducted by the Mathematics Research Center, the University of Wisconsin, Madison, Wis, USA, 1974, pp. 125-145, Academic Press, New York, NY, USA, 1974.

[3] R. Glowinski and O. Pironneau, "Numerical methods for the first biharmonic equation and the two-dimensional Stokes problem," SIAM Review, vol. 21, no. 2, pp. 167-212, 1979.

[4] I. Babuška, J. Osborn, and J. Pitkäranta, "Analysis of mixed methods using mesh dependent norms," Mathematics of Computation, vol. 35, no. 152, pp. 1039-1062, 1980.

[5] R. S. Falk and J. E. Osborn, "Error estimates for mixed methods," RAIRO Analyse Numérique, vol. 14, no. 3, pp. 249-277, 1980.

[6] T. Gudi, "Residual-based a posteriori error estimator for the mixed finite element approximation of the biharmonic equation," Numerical Methods for Partial Differential Equations, vol. 27, no. 2, pp. 315-328, 2011.

[7] T. Gudi, N. Nataraj, and A. K. Pani, "Mixed discontinuous Galerkin finite element method for the biharmonic equation," Journal of Scientific Computing, vol. 37, no. 2, pp. 139-161, 2008.

[8] B. Cockburn, J. Gopalakrishnan, and R. Lazarov, "Unified hybridization of discontinuous Galerkin, mixed, and continuous Galerkin methods for second order elliptic problems," SIAM Journal on Numerical Analysis, vol. 47, no. 2, pp. 1319-1365, 2009.

[9] B. Cockburn, B. Dong, and J. Guzmán, "A hybridizable and superconvergent discontinuous Galerkin method for biharmonic problems," Journal of Scientific Computing, vol. 40, no. 1-3, pp. 141-187, 2009.

[10] F. Brezzi and M. Fortin, Mixed and Hybrid Finite Element Methods, Springer, New York, NY, USA, 1991.

[11] D. N. Arnold and R. Winther, "Mixed finite elements for elasticity," Numerische Mathematik, vol. 92, no. 3, pp. 401-419, 2002.

[12] S. Adams and B. Cockburn, "A mixed finite element method for elasticity in three dimensions," Journal of Scientific Computing, vol. 25, no. 3, pp. 515-521, 2005.

[13] D. N. Arnold, G. Awanou, and R. Winther, "Finite elements for symmetric tensors in three dimensions," Mathematics of Computation, vol. 77, no. 263, pp. 1229-1251, 2008.

[14] J. Guzmán and M. Neilan, "Symmetric and conforming mixed finite elements for plane elasticity using rational bubble functions," Numerische Mathematik. In press.

[15] C. Johnson, "On the convergence of a mixed finite-element method for plate bending problems," Numerische Mathematik, vol. 21, pp. 43-62, 1973.

[16] K. Hellan, Analysis of Elastic Plates in Flexure by a Simplified Finite Element Method, Acta Polytechnica Scandinavica. Civil Engineering and Building Construction Series, Norges Tekniske Vitenskapsakademi, 1967. 
[17] K. Herrmann, "Finite element bending analysis for plates," Journal of the Engineering Mechanics Division, vol. 93, pp. 4983, 1967.

[18] E. M. Behrens and J. Guzmán, "A mixed method for the biharmonic problem based on a system of first-order equations," SIAM Journal on Numerical Analysis, vol. 49, no. 2, pp. 789-817, 2011.

[19] J. Huang, X. Huang, and W. Han, "A new $C^{0}$ discontinuous Galerkin method for Kirchhoff plates," Computer Methods in Applied Mechanics and Engineering, vol. 199, no. 23-24, pp. 1446-1454, 2010.

[20] S. C. Brenner and L. R. Scott, The Mathematical Theory of Finite Element Methods, Springer, New York, NY, USA, 3rd edition, 2008.

[21] P. B. Bochev, C. R. Dohrmann, and M. D. Gunzburger, "Stabilization of low-order mixed finite elements for the Stokes equations," SIAM Journal on Numerical Analysis, vol. 44, no. 1, pp. 82-101, 2006.

[22] J. Li and Y. He, "A stabilized finite element method based on two local Gauss integrations for the Stokes equations," Journal of Computational and Applied Mathematics, vol. 214, no. 1, pp. 58-65, 2008.

[23] P. B. Bochev and C. R. Dohrmann, "A computational study of stabilized, low-order $C^{0}$ finite element approximations of Darcy equations," Computational Mechanics, vol. 38, no. 4-5, pp. 323333, 2006.

[24] K. Nafa, "Local projection finite element stabilization for Darcy flow," International Journal of Numerical Analysis and Modeling, vol. 7, no. 4, pp. 656-666, 2010.

[25] F. Shi, J. Yu, and K. Li, "A new stabilized mixed finiteelement method for Poisson equation based on two local Gauss integrations for linear element pair," International Journal of Computer Mathematics, vol. 88, no. 11, pp. 2293-2305, 2011.

[26] R. An and K. T. Li, "Stabilized mixed finite element approximation for a fourth-order obstacle problem," Acta Mathematicae Applicatae Sinica, vol. 32, no. 6, pp. 1068-1078, 2009.

[27] J. Guzmán and M. Neilan, "Conforming and divergence free stokes elements on general triangularmeshes," Mathematics of Computation. In press.

[28] K. Feng and Z.-C. Shi, Mathematical Theory of Elastic Structures, Springer, Berlin, Germany, 1996.

[29] J. N. Reddy, Theory and Analysis of Elastic Plates and Shells, CRC Press, New York, NY, USA, 2nd edition, 2006.

[30] P. Clément, "Approximation by finite element functions using local regularization," Revue Française d'Automatique, Informatique, Recherche Opérationnelle. Analyse Numérique, vol. 9, no. 2, pp. 77-84, 1975.

[31] M. I. Comodi, "The Hellan-Herrmann-Johnson method: some new error estimates and postprocessing," Mathematics of Computation, vol. 52, no. 185, pp. 17-29, 1989.

[32] M. Dauge, Elliptic Boundary Value Problems on Corner Domains, Springer, Berlin, Germany, 1988.

[33] P. Grisvard, Singularities in Boundary Value Problems, Masson, Paris, France, 1992.

[34] S. C. Brenner, T. Gudi, and L. Sung, "An a posteriori error estimator for a quadratic $C^{0}$-interior penalty method for the biharmonic problem," IMA Journal of Numerical Analysis, vol. 30, no. 3, pp. 777-798, 2010.

[35] M. Wang, "On the necessity and sufficiency of the patch test for convergence of nonconforming finite elements," SIAM Journal on Numerical Analysis, vol. 39, no. 2, pp. 363-384, 2001.
[36] R. Verfürth, A Review of a Posteriori Error Estimation and Adaptive Mesh-Refinement Techniques, Wiley-Teubner, Chichester, $\mathrm{UK}, 1996$. 


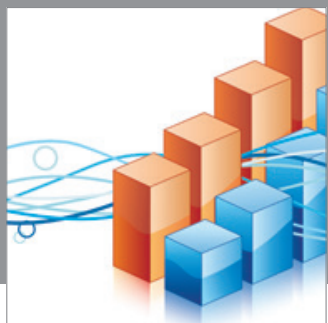

Advances in

Operations Research

mansans

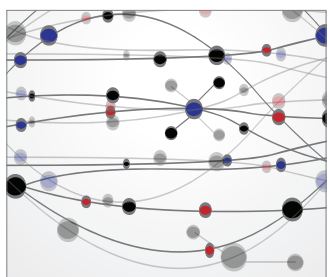

The Scientific World Journal
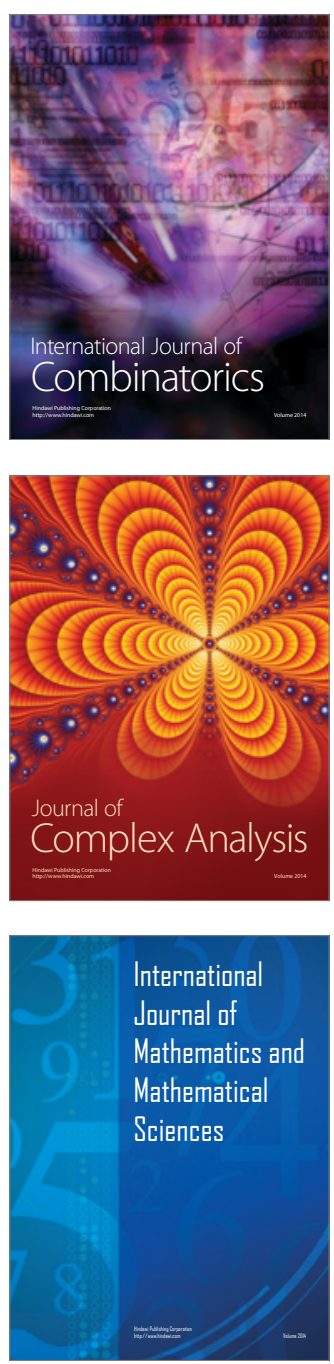
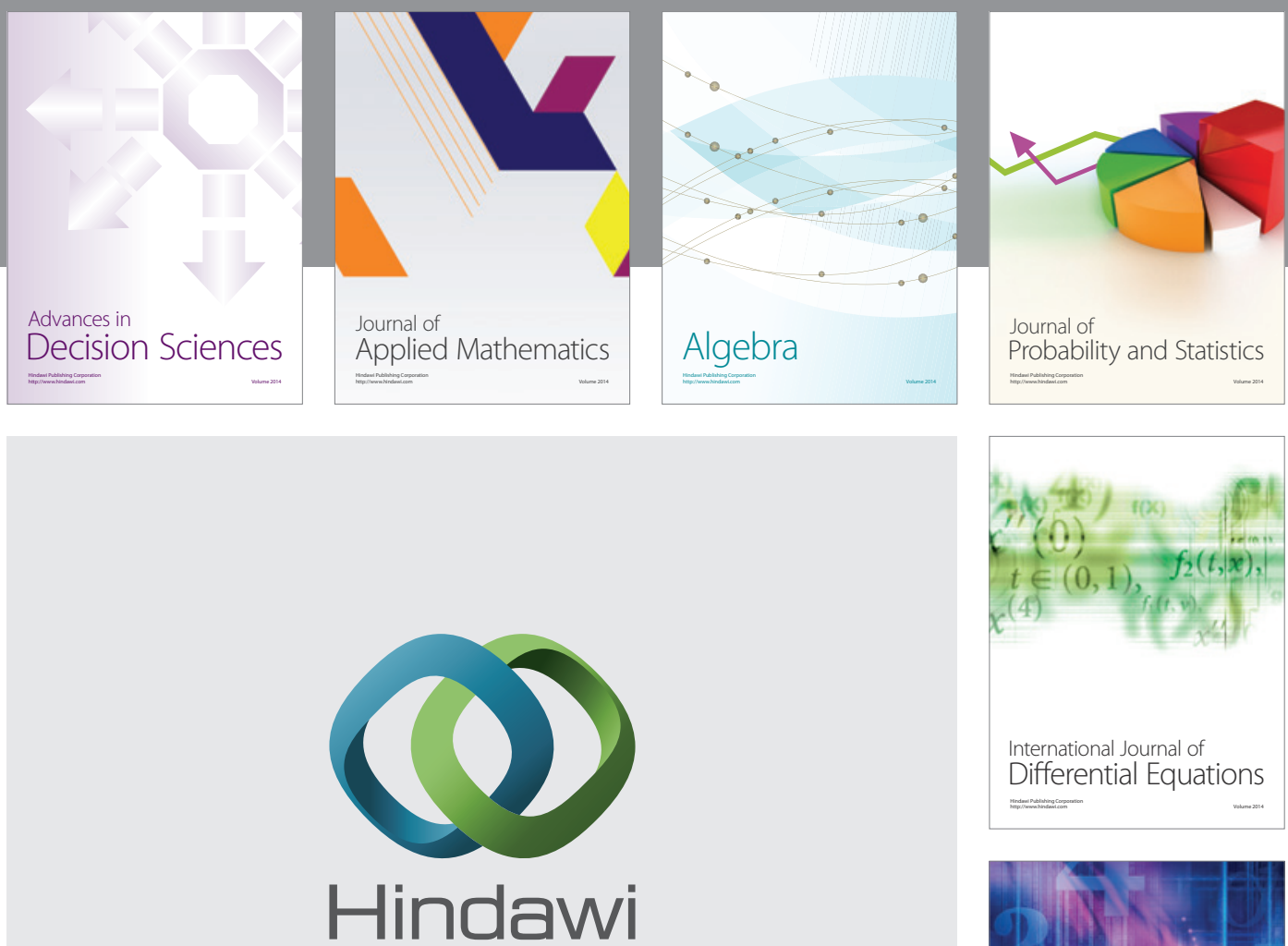

Submit your manuscripts at http://www.hindawi.com
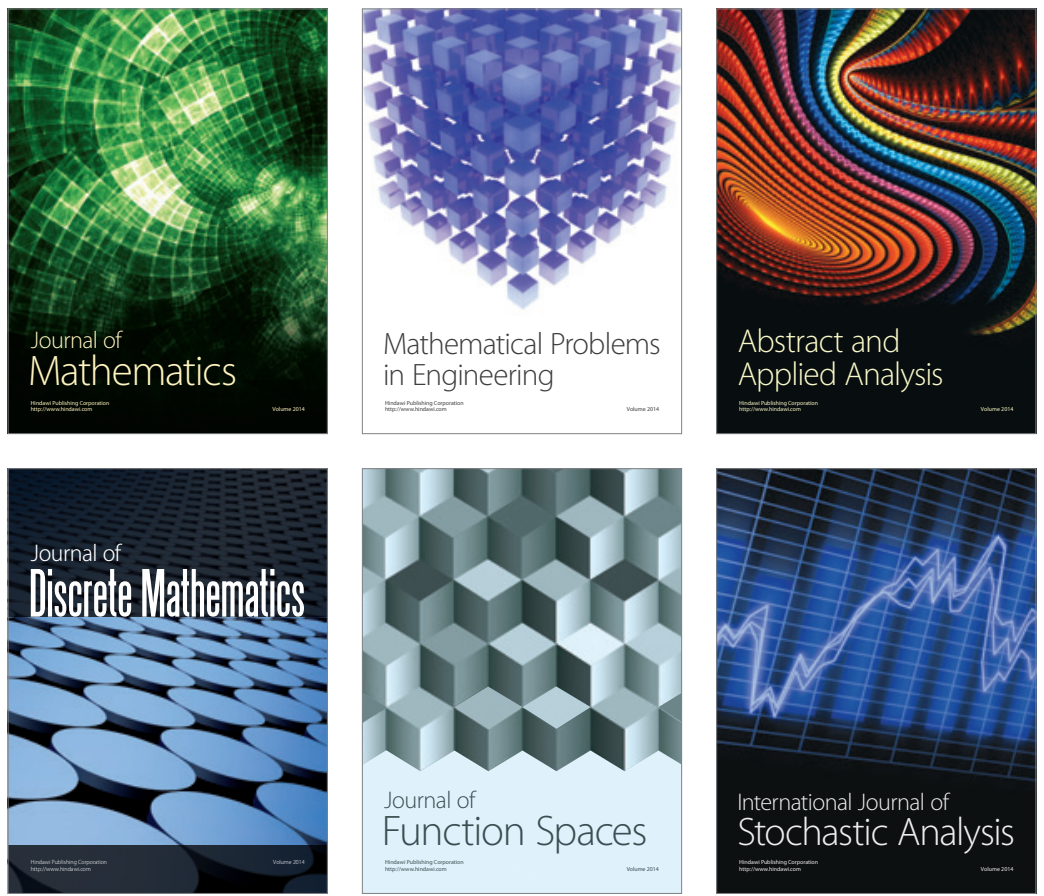

Journal of

Function Spaces

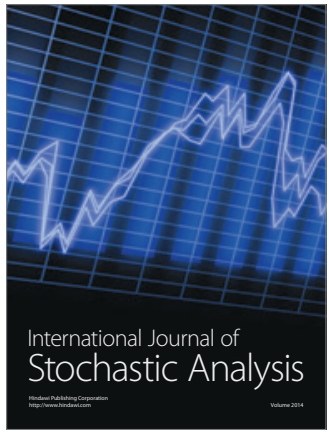

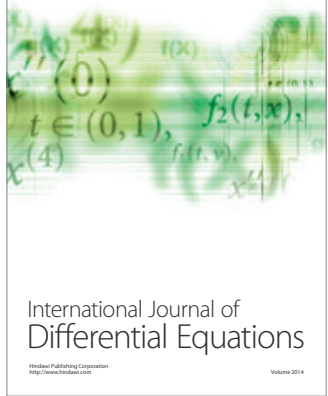
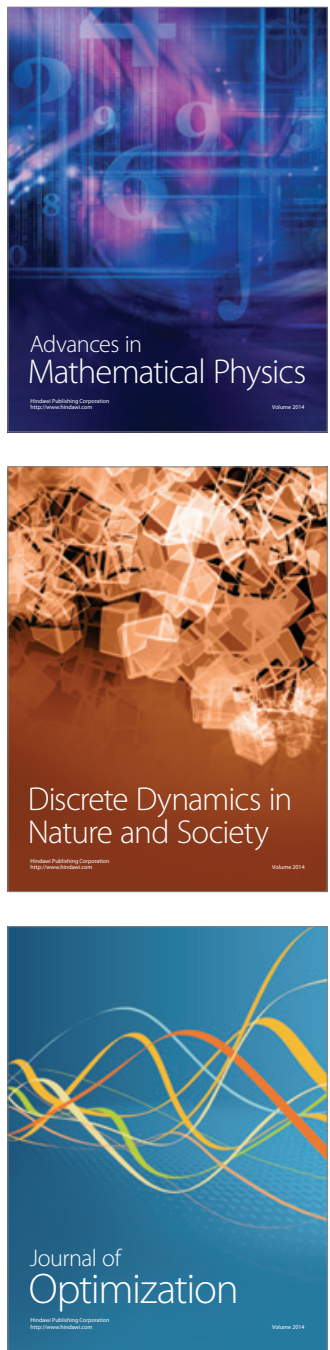